\section{M7 IMPACT AND EVALUATION OF ELECTRONIC CLINICIAN- TO-CLINICIAN ADVICE SERVICE (E- CONSULTATION)}

S Sufyan, E Fearns, MN Khan, G Smith, GL Esterbrook, SL Meghjee, M Thirumaran, J McCreanor, P Blaxill, AOC Johnson, A Dwarakanath. Mid Yorkshire Hospitals NHS Trust, Wakefield, UK

\subsection{6/thoraxjnl-2017-210983.429}

Background Electronic clinician-to-clinician advice service (Econsultation) is a telehealth modality ${ }^{1}$ that enables the primary care clinicians to seek advice from specialists through a shared electronic system (Systm one). This is a mode of non-face to face consult and for less complex cases this service potentially reduces unnecessary clinic referrals and provides an efficient specialist input thus improving patient care. ${ }^{2,3}$ This was first piloted in NHS Yorkshire and Humber in 2012. In agreement with clinical commissioning group (CCG), our trust implemented this in March 2015 and we have evaluated the impact of this service.

Method We retrospectively reviewed all patients who had an e-consultation (March 2015 - January 2017). Patient demographics and clinical information were retrieved from systm one. The referral to clinician response time, content of the referrals, the outcome of the e-consultations and the cost analysis based on nationally agreed tariff (Respiratory treatment code- 340, £23 per e-consultation) was evaluated.

Results 324 patients (63+/-16 years, males- 54\%) had an econsultation. Clinicians completed these referrals in 3 days $(\mathrm{IQR}=1-7$ days, range $=0-32$ days). The content of the e-consultations were classified under five domains- investigations $(n=91,28 \%)$, radiology $(n=114,35 \%)$, medications $(n=32$, $10 \%)$, miscellaneous $(n=6,2 \%)$ and mixed $(n=81,25 \%)$. $63 \%(n=204)$ of the referrals were initiated by the general practitioner, 25\% $(\mathrm{n}=81)$ - practice nurses and 12\% $(\mathrm{n}=39)$ trainees. $32 \% \quad(n=105)$ of the e-consultations were recommended for a formal clinic review. Since implementation, this service has generated over $£ 7000$ to the trust.

Discussion and conclusions This novel service is available for routine, non-urgent specialist advice only and is easy to access. This new approach does not seem to have a significant burden to our other ongoing clinical activities. It provides an opportunity to screen potential formal referrals and identifies the need for specific investigations prior to treatment. However a third of all e-consultations were recommended for a clinic review. Further discussion with the CCG is ongoing to improve the service by having a criteria led referrals and to promote training and awareness of this service.

\section{REFERENCES}

1. L.A. Care Health Plan 2012.

2. Harno et al. Journal of Telemed Telecare 6(5):320-9.

3. Kim et al. Journal of General Internal Medicine 2009;24(5):614-619.
M8

CAN RELIABLE DELIVERING OF THE 48-HOUR ANTIMICROBIAL REVIEW RESULT IN A REDUCTION IN THE NUMBER OF DAYS A PATIENT STAYS ON INTRAVENOUS ANTIBIOTICS ON A RESPIRATORY UNIT? RESULTS FROM A TRAINEE LED QUALITY IMPROVEMENT PROJECT (QIP)

A Asour, S Abburu, J Sood, N Jaafar, D Hobday, S Khan, M Madhani, N Ahmad, A Choudhury. Barking, Havering and Redbridge NHS TRUST, Queen's Hospital, Romford, UK

10.1136/thoraxjnl-2017-210983.430

Introduction and Objectives Effective antimicrobial stewardship aims to reduce healthcare associated infections such as hospital-acquired pneumonia, prevent misuse and overuse of antibiotics, minimise the development of resistance ${ }^{1}$ and shorten hospital stay. The need for antibiotics should be reviewed at 48 hours of starting antibiotic is the focus part of the "start smart then focus" approach. ${ }^{2}$ The aim of our QIP was to improve the percentage completion of the 48 hour antimicrobial review box section of our newly designed inpatient drug charts to over 90\% across two 30-bed acute respiratory wards within 7 weeks.

Methods 30 sequential antibiotic prescriptions were examined twice weekly for a 7 week period (QI Period 10/01/2017-24/ 02/2017). The Outcome measures were: i) 48 hour box ticked ii) Days on IV-antibiotics. Process measures were iii) Signature recorded iv) Date of 48 hour review. The Balancing measures were: v) Indication and vi) Duration of antibiotic recorded. 14 measurement cycles with 7 PDSA interventions took place. Results During the QI period, 334/420 (79\%) of 48 hour Antimicrobial Review boxes were ticked. Commonly ticked options were; Continue Antibiotics $(n=230)$, IV to PO $(n=88)$ and Stop $(n=15)$. Commonest treatment indications were: Community acquired pneumonia (28\%) and lower respiratory tract infection (22\%). We achieved and sustained 100\% completion of our 48 hour review with an associated reduction of IV antibiotic use by mean of 1.1 days (figure 1). 48 hour signature recorded on the drug chart increased from $37 \%$ to 100\%. Balancing measures remained unaffected during QI period.

Conclusions The PDSA Interventions with greatest impact were 1) Educational talk by consultant and 2) Pharmacy engagement to highlight non-completion. The QI methodology can be replicated to other respiratory wards to improve antimicrobial stewardship. Appropriate de-escalation may reduce unnecessary use of IV antibiotics.

\section{REFERENCES}

1. NICE: Antimicrobial stewardship: Systems and processes for effective antimicrobial medicine use. NICE guideline [NG15] Published August, 2015. https://www.nice. org.uk/guidance/ng15

2. Antimicrobial stewardship: Start smart-then focus. Public Health England Updated 25th March, 2015. https://www.gov.uk/government/publications/antimicrobialstewardship-start-smart-then-focus 